\title{
Involvement of transient receptor potential vanilloid 1 channel expression in orofacial cutaneous hypersensitivity following tooth pulp inflammation
}

\author{
Tetsuro Watase1), Kohei Shimizu²,3), Hiroki Komiya1), Kinuyo Ohara2), \\ Koichi Iwata ${ }^{4,5}$, and Bunnai Ogiso ${ }^{2,3)}$ \\ 1)Division of Applied Oral Sciences, Nihon University Graduate School of Dentistry, Tokyo, Japan \\ 2)Department of Endodontics, Nihon University School of Dentistry, Tokyo, Japan \\ 3)Divisions of Advanced Dental Treatment, Dental Research Center, Nihon University School of Dentistry, \\ Tokyo, Japan \\ 4)Department of Physiology, Nihon University School of Dentistry, Tokyo, Japan \\ 5)Division of Functional Morphology, Dental Research Center, Nihon University School of Dentistry, \\ Tokyo, Japan
}

(Received December 12, 2016; Accepted January 5, 2017)

\begin{abstract}
A study was conducted to evaluate the mechanisms underlying ectopic orofacial pain associated with tooth pulp inflammation in rats. We observed a significant decrease in the head withdrawal threshold (HWT) response to mechanical and heat stimuli applied to the ipsilateral facial skin upon application of complete Freund's adjuvant (CFA) to the upper first molar (M1TP) in comparison to application of vehicle. A large number of trigeminal ganglion (TG) neurons showed transient receptor potential vanilloid 1 (TRPV1) immunoreactivity (IR), and some of them were retrogradely labeled with fluorogold injected into the facial skin. A large number of cells showing IR for glial fibrillary acidic protein (GFAP) were observed in the 2nd compared to the 1st or 3rd branch regions of the TG, and TG cells innervating the facial skin were also surrounded by GFAP-IR cells. After administration of TRPV1 antagonist into the facial skin of M1TP CFA-treated
\end{abstract}

Correspondence to Dr. Kohei Shimizu, Department of Endodontics, Nihon University School of Dentistry, 1-8-13 Kanda-Surugadai, Chiyoda-ku, Tokyo 101-8310, Japan

E-mail: shimizu.kouhei01@nihon-u.ac.jp

J-STAGE Advance Publication: February 26, 2018

Color figures can be viewed in the online issue at J-STAGE.

doi.org/10.2334/josnusd.16-0854

DN/JST.JSTAGE/josnusd/16-0854 rats, the decrease of HWTs in response to mechanical and heat stimulation of the facial skin was significantly reversed. The present findings suggest that the excitability of TG neurons is enhanced upon tooth pulp inflammation, leading to overexpression of TRPV1 in TG neurons innervating the facial skin, and that satellite glial cells are also activated, resulting in the development of ectopic orofacial pain.

Keywords: ectopic pain; pulpitis; trigeminal ganglion; trigeminal nerve; satellite glial cells.

\section{Introduction}

It is well established that ectopic orofacial pain and nonodontogenic toothache often occur in association with tooth pulp inflammation $(1,2)$. As such pain symptoms may lead to misdiagnosis and inappropriate treatment of patients with toothache $(3,4)$, it is important to clarify the mechanisms responsible for this type of ectopic orofacial pain and non-odontogenic toothache.

Tooth pulp inflammation triggers activation of the trigeminal ganglion (TG) neurons, generating a barrage of action potentials that leads to their sensitization $(5,6)$. Sensitized TG neurons then produce various molecules that are released within the TG, resulting in activation of satellite glial cells (SGCs), followed by spreading 
activation of glial cells within the TG $(5,6)$. Glial cell activation within the TG is believed to be involved in the mechanisms underlying ectopic orofacial pain associated with tooth pulp inflammation (5). However, details of these mechanisms remain unclear.

The transient receptor potential vanilloid 1 (TRPV1) channel is known to be one of the key molecules involved in the hyperexcitation of primary afferent neurons associated with peripheral inflammation (7-11). TRPV1 is an ionotropic heat-sensing receptor channel widely expressed in C-fiber primary afferents (9). It has been reported that within the TG, TRPV1 plays a pivotal role in modulating the excitability of $\mathrm{C}$-fiber afferents following tissue inflammation $(5,7)$. Noxious heat stimuli $\left(>43^{\circ} \mathrm{C}\right)$ activate the TRPV1 channel, and noxious information is transmitted from primary afferent neurons to secondary neurons $(12,13)$. A number of previous studies have also reported that TRPV1 is expressed in TG neurons following orofacial inflammation, suggesting its involvement in the development of orofacial hyperalgesia $(5,7,14)$. It is highly possible that TRPV1 expression is upregulated in TG neurons following tooth pulp inflammation and that this channel protein is also involved in orofacial ectopic pain. It has also been reported that SGCs are activated in the TG following tooth pulp inflammation, and that these cells are directly involved in the spread of neuronal excitability within the TG (5). Therefore, we have hypothesized that the excitability of TG neurons is enhanced following tooth pulp inflammation as a result of TRPV1 overexpression in TG neurons innervating the facial skin and activation of SGCs, leading to ectopic orofacial pain. To test this hypothesis, we analyzed nocifensive reflex responses evoked by mechanical and heat stimulation, expression of TRPV1 and glial fibrillary acidic protein (GFAP) in the TG after tooth pulp inflammation, and the effect of a TRPV1 antagonist on the nocifensive reflex.

\section{Materials and Methods}

\section{Animals}

Male Sprague-Dawley rats ( $n=59$, Japan SLC, Hamamatsu, Japan) weighing between 250 and $350 \mathrm{~g}$ were used for the present study. They were housed in an animal room set at a temperature of $23^{\circ} \mathrm{C}$ with a $12 / 12 \mathrm{~h}$ light/ dark cycle under pathogen-free conditions. Food and water were provided ad libitum. The experimental study was ethically approved by the Animal Experimentation Committee of Nihon University School of Dentistry (AP14D032, AP14D001-3) and conducted in accordance with the guidelines of the International Association for the Study of Pain (15). The number of rats used in the study was calculated based on the minimum requirement for statistical analysis.

\section{Application of complete Freund's adjuvant (CFA) to the tooth pulp}

Rats were initially anesthetized lightly with $2 \%$ isoflurane (Mylan, Canonsburg, PA, USA) and then deeply by intraperitoneal (i.p.) administration of sodium pentobarbital (50 mg/kg; Schering Plough, Whitehouse Station, NJ, USA). A warm mat (set at $37^{\circ} \mathrm{C}$ ) to facilitate heat retention was used, and the animals were positioned supine for administration of CFA (Sigma-Aldrich, St. Louis, MO, USA). Rats received 50\% CFA (diluted in saline) or vehicle (isotonic saline) into the unilateral upper first molar tooth pulp (M1TP). After gently opening the mouth, the M1TP was exposed by means of a low-speed dental drill using a round tungsten carbide bur under water-cooling. A small dental paper point (diameter, 0.15 $\mathrm{mm}$; length, $1.5 \mathrm{~mm}$ ), previously soaked in either CFA or vehicle solution, was then placed onto the exposed M1TP. Sealing of the exposed pulp with dental cement was carried out just prior to the experiment $(n=6$ per group).

\section{Measurement of the head-withdrawal reflex threshold in response to heat or mechanical stimulation}

Under light anesthesia with isoflurane in oxygen, vehicle or CFA solution was applied to the M1TP after exposure. Starting at $24 \mathrm{~h}$ after drug administration, the change in the head-withdrawal reflex threshold (HWT) response to heat stimulation was monitored. For reflex electromyogram (EMG) recording (inter-electrode distance, 5 to 6 $\mathrm{mm}$ ), the lateral face hair was shaved and bipolar enamelcoated stainless steel wire electrodes (Narishige, Tokyo, Japan) were inserted into the splenius capitis muscles. Following the application of CFA or vehicle solution, the rats were lightly anesthetized with $2 \%$ isoflurane, a contact heat probe $\left(9 \mathrm{~mm}^{2}\right.$; Intercross, Tokyo, Japan) was placed on the facial skin, and graded heat stimuli $\left(35-60^{\circ} \mathrm{C} ; 1^{\circ} \mathrm{C} / \mathrm{s}\right.$; cutoff, $\left.60^{\circ} \mathrm{C}\right)$ were applied. The heat HWT was determined as the temperature threshold required to evoke EMG activity following heat stimulation of the facial skin. Rats were stimulated three times with an interval of $5 \mathrm{~min}$ between stimulations, and the average heat HWT was calculated. The baseline HWT was determined prior to the application of CFA or vehicle. On days 1 and 3 after application of CFA or vehicle to the M1TP, changes in the heat HWT values were determined ( $n=6$ in each group). The von Frey filaments (Touch Test Sensory Evaluator; North Coast Medical, Morgan Hill, CA, USA) were used to measure the mechanical HWT of the facial skin ipsilateral to the vehicle or CFA 
application. Upon application of mechanical stimuli, rats were held in a tube and were able to escape from the stimulus at any time. The probing filament was applied to the lateral facial skin, and the HWT response to mechanical stimuli was measured. Testing with each von Frey filament was performed 3 times with intervals of a few seconds. The mechanical HWT in each trial was determined as the minimum pressure intensity needed to evoke head withdrawal in response to more than three of five stimuli. Rats were tested at intervals of $5 \mathrm{~min}$ to prevent frequent stimuli inducing hypersensitivity of the facial skin. On days 1 and 3 after application of CFA or vehicle to the M1TP, changes in the mechanical HWT were also monitored ( $n=6$ per group). All behavioral tests were conducted during the light phase of the light/ dark cycle.

\section{TRPV1 and GFAP immunohistochemistry in combination with a retrograde tracer}

To assess the expression of TRPV1 or GFAP in TG neurons innervating the facial skin, $5.0 \mu \mathrm{L}$ of $10 \%$ fluorogold (FG, Fluorochrome, Denver, CO, USA) dissolved in saline was applied to the facial skin ipsilateral to the M1TP. Light anesthesia was induced with $2 \%$ isoflurane, and then rats were deeply anesthetized with intraperitoneal (i.p.) administration of sodium pentobarbital (50 $\mathrm{mg} / \mathrm{kg}$; Schering Plough) to apply CFA or vehicle to the M1TP $72 \mathrm{~h}$ after application of FG to the facial skin. Transcardiac perfusion with saline solution followed by a fixative containing $4 \%$ paraformaldehyde (PFA) in $0.1 \mathrm{M}$ phosphate buffer ( $\mathrm{pH}$ 7.4) was performed under sodium pentobarbital anesthesia $(100 \mathrm{mg} / \mathrm{kg}$, i.p., $n=6)$. The ipsilateral TGs were then removed and fixed in $4 \%$ PFA for $24 \mathrm{~h}$ at $4^{\circ} \mathrm{C}$. The specimens were then transferred to $20 \%$ sucrose $(\mathrm{w} / \mathrm{v})$ in distilled water for several days, subsequently embedded in Tissue Tek (Sakura Finetek, Torrance, CA, USA), and stored until cryosectioning at $-20^{\circ} \mathrm{C}$. Sections $10 \mu \mathrm{m}$ thick were cut in the horizontal plane along the longitudinal axis of the TG. Every eighth section was thaw-mounted on a microslide glass (MAS-GP type A, Matsunami, Kishiwada, Japan) and dried overnight at room temperature. Four sections were chosen from each collected TG, and further processed for TRPV1 or GFAP immunohistochemistry. Sections were incubated with rabbit anti-TRPV1 polyclonal antibody (1:200; Abcam, Cambridge, MA, USA) or mouse antiGFAP polyclonal antibody (1:800; Sigma-Aldrich) after dilution in $0.01 \mathrm{M}$ PBS containing 4\% normal goat serum (NGS) and $0.3 \%$ Triton X-100 (Sigma-Aldrich) for 72 $\mathrm{h}$ at $4^{\circ} \mathrm{C}$. After washing with $0.01 \mathrm{M}$ PBS, the sections were incubated in Alexa Fluor 488 goat anti-rabbit IgG or
Alexa Fluor 488 goat anti-mouse $\operatorname{IgG}$ (1:300; Invitrogen, Paisley, UK) for $2 \mathrm{~h}$ at room temperature. After rinsing in $0.01 \mathrm{M}$ PBS, the sections were cover-slipped with mounting medium (Thermo Fisher Scientific, Fremont, CA, USA) and examined using a BZ-9000 fluorescence microscope (Keyence, Osaka, Japan). No non-specific signal was detected in the absence of primary antibody. The following formula was used to analyze the number of TRPV1-IR cells in the TG of the 2nd branch region was counted for each rat (Sensiv Measure; Mitani, Fukui, Japan; $n=6$ in each group): (TRPV1 + FG-IR cells/ FG-IR cells) $\times 100 \%$.

\section{Administration of TRPV1 antagonist into the skin overlying the masseter muscle}

The TRPV1 antagonist, N-(3-methoxyphenyl)-4-chlorocinnamide (SB366791; Sigma-Aldrich), was dissolved in 50:50 dimethyl sulfoxide: saline. Light anesthesia was induced with 2\% isoflurane, and then SB366791 was applied to the facial skin on day 1 after CFA application. HWT in response to heat or mechanical stimulation was measured at 10, 30, 60, 90, and 120 min after SB366791 application.

\section{Statistical analysis}

Data are presented as mean \pm standard error of the mean (SEM). HWT in response to heat or mechanical stimulation at each time point was subjected to two-way analysis of variance (ANOVA) followed by Tukey's post $h o c$ test. Student's $t$-test was used to compare the number of TRPV1-IR and GFAP-IR cells between vehicle- and CFA-treated rats. Similarly, HWT in response to heat or mechanical stimulation after SB366791 administration at each time point was subjected to two-way ANOVA followed by Tukey-Kramer's post hoc test or Tukey's post hoc test. Differences were considered statistically significant at $P<0.05$.

\section{Results}

\section{Change in HWT in response to heat or mechanical} stimulation following M1TP inflammation

HWT of the ipsilateral facial skin in response to mechanical stimulation was significantly decreased on day 1 after application of CFA (post CFA) to the M1TP relative to vehicle-applied rats or pre-application baseline values (Vehicle: $P<0.01$, vs. pre CFA: $P<0.05 ; n=6$ in each group) (Fig. 1A). HWT of the ipsilateral facial skin in response to heat was also significantly lower on days 1 to 3 after M1TP CFA application relative to vehicle-applied rats or pre-application baseline values (Vehicle: $P<0.05$, vs. pre CFA: $P<0.001 ; n=6$ in each group) (Fig. 1B). 

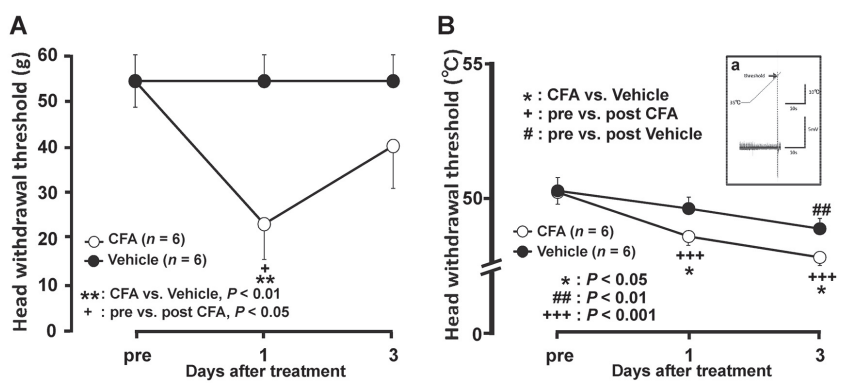

Fig. 1 Mechanical or heat hyperalgesia of the facial skin following application of CFA to M1TP. A: Change in HWT of the ipsilateral facial skin in response to mechanical stimulation following application of CFA to the M1TP. (CFA: $n=6$, vehicle: $n=6$, two-way ANOVA with repeated measures, followed by Tukey's post hoc test). B: Change in HWT of the ipsilateral facial skin in response to heat stimulation following application of CFA to the M1TP. (CFA: $n=6$, vehicle: $n$ $=6$, two-way ANOVA with repeated measures, followed by Tukey's post hoc test). Ba: Example of an EMG recording from the splenius capitis muscles during heat stimulation of the facial skin. Data are presented as mean \pm standard error of the mean (SEM). *: CFA vs. vehicle, + : pre vs. post CFA, \#: pre vs. post vehicle, $+: P<0.05,{ }^{* *}: P<0.01$, \#\#: $P<0.01,+++: P<0.001$.
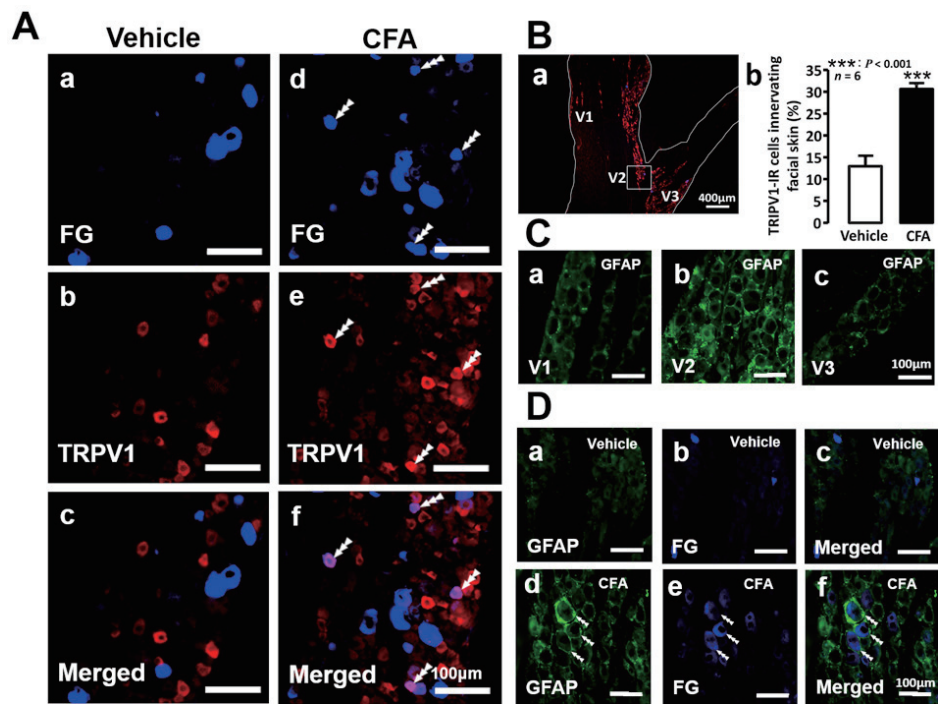

Fig. 2 FG labeling of TRPV1- and GFAP-IR TG neurons after M1TP inflammation. Aa-f: TRPV1-IR cells labeled with FG in rats treated with vehicle or CFA. The arrowheads indicate the co-expression of FG-labeled and TRPV1-IR cells in CFA-treated rats (Ad-Af). Ba: The 1st (V1), 2nd (V2) and 3rd (V3) regions of the TG. The 2nd region is indicated by the square box. $\mathrm{Bb}$ : The mean relative proportion of FG-labeled TRPV1-IR cells in the 2nd branch region in rats subjected to M1TP treatment with CFA or vehicle (CFA: $n=6$, vehicle: $n=6$, Student's $t$-test). Ca-Cc: GFAP-IR cells in the 1st $(\mathrm{Ca}), 2 \mathrm{nd}(\mathrm{Cb})$ and $3 \mathrm{rd}(\mathrm{Cc})$ regions in the TG of rats subjected to M1TP treatment with CFA. Da-c: GFAP-IR cells in the TG of rats subjected to M1TP treatment with vehicle. Dd-f: GFAP-IR cells in the TG of rats subjected to M1TP treatment with CFA. The arrowheads indicate co-expression of GFAP-IR and FG-labeled cells in CFA-treated rats (Dd-Df). A, C and D: Scale bars in the panels indicate $100 \mu \mathrm{m}$. B: Scale bars in the panels indicate $400 \mu \mathrm{m} . * * *$ : Vehicle vs. CFA, $P<0.001$ (Vehicle vs. CFA).

HWT in response to heat was also significantly reduced at 3 days following the application of vehicle solution to the M1TP compared with the pre-application baseline value $(P<0.01)$ (Fig. 1B). On the other hand, we failed to observe any significant changes in HWT in response to mechanical stimulation in vehicle-injected rats over the 3 days observation period (Fig. 1 A).

\section{TRPV1 expression in GFAP-IR cells in the} trigeminal ganglion upon M1TP inflammation

As a result, a large number of TG neurons showed TRPV1-IR, and some of them were retrogradely labeled with FG injected into the facial skin (Fig. 2Aa-f). A large number of FG-labeled TRPV1-IR neurons were observed in the 2 nd branch region of the trigeminal nerve, as 


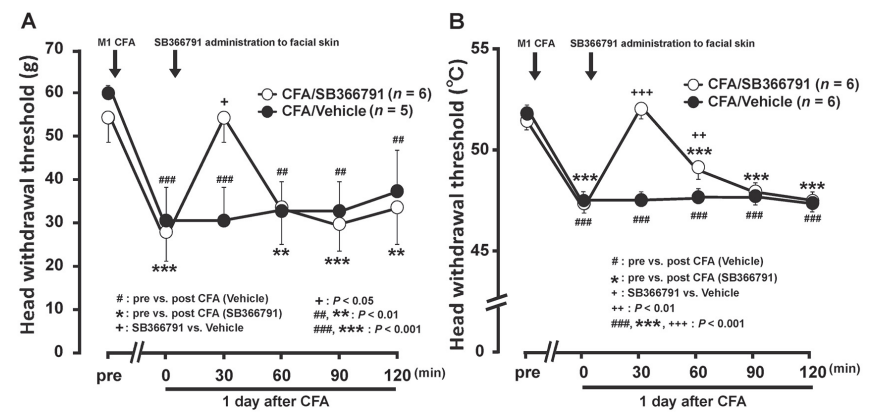

Fig. 3 Effect of TRPV1 antagonist on HWT in response to heat or mechanical stimulation. A: Effect of SB366791 on HWT in response to mechanical stimulation of the ipsilateral facial skin following application of CFA to the M1TP. (CFA/SB366791: $n=6$, CFA/vehicle: $n=5$, two-way ANOVA followed by Tukey-Kramer's post hoc test). B: Effect of SB366791 on HWT in response to heat stimulation of the ipsilateral facial skin following application of CFA to the M1TP. (CFA/ SB366791: $n=6$, CFA/vehicle: $n=6$, two-way ANOVA followed by Tukey's post hoc test). \#: pre vs. post CFA (Vehicle), *: pre vs. post CFA (SB366791), +: SB366791 vs. Vehicle, $+: P<$ $0.05, \# \#, * *,++: P<0.01, \# \#$, ***, $+++: P<0.001$.

indicated by the square box in Fig. 2Ba. The proportion of FG-labeled TRPV1-IR cells in the 2nd branch region was significantly higher in M1TP CFA-applied rats than in M1TP vehicle-applied rats $(P<0.001, n=6)$ (Fig. $2 \mathrm{Bb}$ ). We also observed a large number of GFAP-IR cells in the 2nd (Fig. 2Cb) compared to the 1st (Fig. 2Ca) or 3rd (Fig. 2Cc) regions of the TG. We failed to observe any GFAP-IR cells in M1TP vehicle-applied rats (Fig. 2Da-c). A large number of TG neurons labeled with FG injected into the facial skin were surrounded by GFAP-IR cells in the 2 nd branch region of the TG (Fig. 2Dd-f).

\section{Effect of TRPV1 antagonist on HWT induced by heat or mechanical stimulation}

Upon administration of SB366791, a TRPV1 antagonist, into the facial skin of M1TP CFA-applied rats, the decreases in HWT in response to mechanical stimulation were significantly reversed at $30 \mathrm{~min}$ following drug injection relative to those of vehicle-applied rats $(P<$ 0.05; CFA/Vehicle, $n=5$; CFA/SB366791, $n=6$ ) (Fig. 3A). Similarly, the decreases in HWT in response to heat stimulation of the facial skin in CFA-applied rats were significantly reversed at 30 and 60 min after SB366791 administration crelative to vehicle-injected rats (at 30 $\min , P<0.001$; at $60 \mathrm{~min}, P<0.01$; CFA/Vehicle, $n=6$; CFA/SB366791, $n=6$ ) (Fig. 3B).

\section{Discussion}

When tooth pulp becomes inflamed, ectopic orofacial pain, as well as a toothache, is known to occur frequently $(1,4,16)$. Radiating pain diffusing to the distant tooth or facial skin sometimes leads to misdiagnosis or inappropriate treatment. It is therefore essential to clarify the mechanisms underlying this type of pain. In the present study, behavioral and immunohistochemical experiments were conducted using rats with CFA-induced tooth pulp inflammation in an attempt to clarify some of the mechanisms underlying ectopic orofacial pain.

It is well established that application of CFA to tooth pulp enhances the excitability of TG neurons innervating both the inflamed and non-inflamed tooth pulp, resulting in ectopic pain in the adjacent tooth (5). There are several lines of evidence to suggest that hyperexcitability of TG neurons leads to produce and release various molecules such as toll-like receptor 4 (TLR4), nitric oxide, nerve growth factor, substance P, ATP and calcitonin gene-related peptide in these neurons, resulting in enhancement of TG neuronal activity in non-inflamed orofacial tissues. It has also been reported that satellite glial cells (SGCs) are activated in a broader area within the TG after nerve injury or tooth pulp inflammation, and involved in modulating the activity of TG neurons innervating non-inflamed areas of the orofacial region (5). Here we demonstrated a significant decrease of HWT in response to heat and mechanical stimulation in the noninflamed facial skin after M1TP inflammation. These results, together with the previous data, suggest that the mechanisms of non-inflammatory facial pain associated with tooth pulp inflammation involve neuron-neuron or neuron-glia interaction.

The TRPV1 ion channel is known to be expressed predominantly in C-fibers and involved in noxious heat sensory processing in the peripheral nervous system $(10,12)$. When orofacial inflammation occurs, TRPV1 channel expression is enhanced in TG neurons associated with enhancement of p38 phosphorylation, resulting in 
the development of orofacial heat hyperalgesia $(17,18)$. The TRPV1 channel also has various functional interactions with ligand-gated receptors in C-fiber terminals, such as the prostaglandin E receptor, serotonin receptor and bradykinin receptor, and is involved in inflammatory pain processing (18-20). Recent studies have also shown that NGF is expressed in TG neurons following lower lip inflammation and released from TG neurons innervating the inflamed lower lip. NGF plays a role in enhancing the excitability of TG neurons innervating the noninflamed upper lip through overexpression of TRPV1 (21). The TLR4 expression is also induced in TG neurons innervating non-inflamed tongue tissues in the presence of tooth pulp inflammation (6). Furthermore, SGCs are known to be activated within the TG upon tooth pulp inflammation, and this activation spreads over the TG, extending to another branch region of the trigeminal nerve (5). We observed a significant increase of TRPV1 expression in TG neurons innervating the non-inflamed facial skin, and also found that a large number of such neurons were surrounded by GFAP-IR cells.

Overall, our findings suggest that TRPV1 expression in TG neurons innervating the non-inflamed facial skin is involved in the hypersensitization of facial skin associated with tooth pulp inflammation, resulting in the development of ectopic orofacial pain.

\section{Acknowledgments}

This study was supported in part by JSPS KAKENHI Grant Numbers 25462968 and 16K11566.

\section{Conflict of interest}

The authors have no conflict of interest to declare.

\section{References}

1. Bender IB (2000) Pulpal pain diagnosis--a review. J Endod 26, 175-179.

2. Owatz CB, Khan AA, Schindler WG, Schwartz SA, Keiser K, Hargreaves KM (2007) The incidence of mechanical allodynia in patients with irreversible pulpitis. J Endod 33, $552-556$

3. Glick DH (1962) Locating referred pulpal pains. Oral Surg Oral Med Oral Pathol 15, 613-623.

4. Yatani H, Komiyama O, Matsuka Y, Wajima K, Muraoka W, Ikawa M et al. (2014) Systematic review and recommendations for nonodontogenic toothache. J Oral Rehabil 41, 843-852.

5. Matsuura S, Shimizu K, Shinoda M, Ohara K, Ogiso B, Honda K et al. (2013) Mechanisms underlying ectopic persistent tooth-pulp pain following pulpal inflammation. PLoS
One 8, e52840

6. Ohara K, Shimizu K, Matsuura S, Ogiso B, Omagari D, Asano $\mathrm{M}$ et al. (2013) Toll-like receptor 4 signaling in trigeminal ganglion neurons contributes tongue-referred pain associated with tooth pulp inflammation. J Neuroinflammation 10, 139.

7. Numazaki M, Tominaga M (2004) Nociception and TRP channels. Curr Drug Targets CNS Neurol Disord 3, 479-485.

8. Tominaga M, Caterina MJ (2004) Thermosensation and pain. J Neurobiol 61, 3-12.

9. Barabas ME, Stucky CL (2013) TRPV1, but not TRPA1, in primary sensory neurons contributes to cutaneous incisionmediated hypersensitivity. Mol Pain 9, 9.

10. Urata K, Shinoda M, Honda K, Lee J, Maruno M, Ito R et al. (2015) Involvement of TRPV1 and TRPA1 in incisional intraoral and extraoral pain. J Dent Res 94, 446-454.

11. Yang K (2016) Postnatal excitability development and innervation by functional transient receptor potential vanilloid 1 (TRPV1) terminals in neurons of the rat spinal sacral dorsal commissural nucleus: an electrophysiological study. Mol Neurobiol 53, 6033-6042.

12. Caterina MJ, Schumacher MA, Tominaga M, Rosen TA, Levine JD, Julius D (1997) The capsaicin receptor: a heatactivated ion channel in the pain pathway. Nature 389, 816-824.

13. Tominaga $M$, Tominaga $T$ (2005) Structure and function of TRPV1. Pflugers Arch 451, 143-150.

14. Ro JY, Lee JS, Zhang Y (2009) Activation of TRPV1 and TRPA1 leads to muscle nociception and mechanical hyperalgesia. Pain 144, 270-277.

15. Zimmermann M (1983) Ethical guidelines for investigations of experimental pain in conscious animals. Pain 16, 109-110.

16. Wright EF (2008) Pulpalgia contributing to temporomandibular disorder-like pain: a literature review and case report. J Am Dent Assoc 139, 436-440.

17. Thalakoti S, Patil VV, Damodaram S, Vause CV, Langford LE, Freeman SE et al. (2007) Neuron-glia signaling in trigeminal ganglion: implications for migraine pathology. Headache 47, 1008-1023.

18. Kristiansen KA, Edvinsson L (2010) Neurogenic inflammation: a study of rat trigeminal ganglion. J Headache Pain 11, 485-495.

19. Ma W, St-Jacques B, Rudakou U, Kim YN (2017) Stimulating TRPV1 externalization and synthesis in dorsal root ganglion neurons contributes to PGE2 potentiation of TRPV1 activity and nociceptor sensitization. Eur J Pain 21, 575-593.

20. Mathivanan S, Devesa I, Changeux JP, Ferrer-Montiel A (2016) Bradykinin induces TRPV1 exocytotic recruitment in peptidergic nociceptors. Front Pharmacol 7, 178.

21. Shinoda M, Asano M, Omagari D, Honda K, Hitomi S, Katagiri A et al. (2011) Nerve growth factor contribution via transient receptor potential vanilloid 1 to ectopic orofacial pain. J Neurosci 31, 7145-7155. 